\title{
ON THE GROUPS
}

\section{WHICH ARE THE DIRECT PRODUCTS OF TWO SUBGROUPS*}

BY

\section{G. A. MILLER}

If a group is generated by two self-conjugate subgroups which have only identity in common it is said to be the direct product $\dagger$ of these subgroups. It is well known that every operator of one of these groups is commutative with every operator of the other. + Evidently one of the necessary conditions that a group $(g)$ may be the direct product of two subgroups is that it contains a subgroup $\left(g_{1}\right)$ such that every operator of $g_{1}$ is transformed into its various conjugates under $g$ by the operators of $g_{1}$; $i$. e., each operator of $g_{1}$ has in all the same transforms with respect to its own operators as it has with respect to the operators of $\mathrm{g}$. That this condition is not sufficient follows from the cyclical groups whose order is a power of a prime, from the quaternion group, and from many other known groups. This condition is explicitly employed in theoremIII.

Theorem I.-If $\mathrm{g}$ has a solvable quotient group $\mathrm{g} / \mathrm{As}$ such that in the isomorphism of $\mathrm{g}$ with $\mathrm{g} / \mathrm{s}$ to each operator of $\mathrm{g} / \mathrm{st}$ there corresponds one and only one operator of $g$ whose order is a divisor of the order of $g /$ s then $g$ is the direct product of $\&$ and a subgroup which is simply isomorphic to $\mathrm{g} / \mathrm{Af}$.

Let $1, \mathcal{H}_{1}, \mathcal{H}_{2}, \cdots, \mathcal{H}_{m} \equiv \mathcal{G} / \mathcal{A}$ be a series of groups such that each one includes the one which precedes it and some additional operators, each is selfconjugate in $\mathcal{A}_{m}$, and all the quotient groups $\mathcal{H}_{a} / \mathcal{H}_{a_{-1}}(a=1,2, \ldots, m)$ are abelian. $\S$ Let $1, T_{2}, T_{3}, \ldots, T_{n}$ be all the operators of $g$ whose orders are divisor of the order $\left(h_{m}\right)$ of $\mathrm{g} / \mathrm{s} ; n=h_{m}$. It is only necessary to prove that these $n$ operators constitute a group. $\|$ In the isomorphism of $G$ and $G / \mathcal{A}$ each one of these T's evidently corresponds to an operator of the same order

* Presented to the Society at the Columbus meeting August 26, 1899 . Received for publication December 4, 1899.

† As substitution groups these groups have been known for a long time. They occupy a prominent place in the theory of intransitive groups. HöLDER seems to have been the first to call them (as abstract groups) direct products, Mathematische Annalen, vol. 43, p. 330, 1893.

‡ Dyck, Mathematische Annalen, vol. 22, p. 79, 1883.

\& JoRdan, Traité des substitutions, p. 395, 1870.

|| DYck, l. c. 
in $g_{/} / \mathcal{A}$ and all the T's must transform each other in exactly the same manner as the corresponding operators of $g_{/} / \mathcal{L}$ transform each other. Since $\mathcal{A}_{1}$ is abelian the corresponding $T$ 's $\left(1, T_{2}, \cdots T_{h_{1}}\right)$ constitute an abelian group. This group (I) is a self-conjugate subgroup of $q$ since $\mathbb{S}_{1}$ is self-conjugate in $\mathrm{g} / \mathrm{J}$. This proves the theorem if $m=1$.

When $m>1$ we can readily prove the theorem by induction. Suppose that all the $T$ 's which correspond to $\mathscr{H}_{a}(a<m)$ constitute a self-conjugate subgroup $\left(\Im^{a}\right)$ of $q$ and let $T_{a+1}$ be any one of the given $n T$ 's that corresponds to an operator of $\mathrm{HF}_{a+1}$ but is not contained in $\mathrm{Y}^{a} . T_{a+1}$ and $\mathrm{F}^{a}$ clearly generate a group whose order is a divisor of $n$. This group $\mathfrak{J}^{a+1}$ must be transformed into itself by all the operators of $q$ which correspond to $\mathcal{A}_{\alpha+1}$ since $\mathcal{A}_{\alpha+1} / \mathcal{A} \mathcal{A}_{\alpha}$ is abelian. If the order of $z^{a+1}$ is less than that of $\mathcal{S}_{a+1}$ we let $T_{\beta}$ be any one of a given $n T$ 's which corresponds to an operator of $\mathcal{A}_{\alpha+1}$ and is not contained in $\mathfrak{Y}^{a+1}$, $T_{\beta}$ and $r^{a+1}$ will clearly generate a larger group whose order is a divisor of $n$ and which is self-conjugate in the subgroup of $q$ which corresponds to $\mathscr{A}_{a_{+1}}$. Hence we observe, by induction, that all the $T$ "s which correspond to $\mathscr{F}_{a_{+1}}$ constitute a self-conjugate subgroup of $g$ provided all those which correspond to $\mathcal{S F}_{\alpha}$ constitute such a self-conjugate subgroup. We proved above that all those which correspond to $\mathcal{A}_{1}$ constitute such a self-conjugate subgroup. Hence the proof is complete.

Theorem II.-If the order of a group $\mathcal{K}$ is $m n$ ( $m$ and $n$ being prime to each other), and if $\mathcal{K}$ contains a subgroup $m$ of order $m$ which has the property that for every operator $K$ of $\mathcal{K}$ there is an operator $M^{\prime}$ of $m$ such that for every operator $M$ of $m$ the transforms by $K$ and by $M^{\prime}$ are equal,

$$
\left(K^{-1} M K=M^{\prime-1} M M^{\prime}\right),
$$

and if the quotient group $\mathcal{K} / \mathrm{m}$ is solvable, then $\mathcal{K}$ is the direct product of its subgroups of orders $m$ and $n$ respectively.

It is clear that $m$ is a self-conjugate subgroup of $\mathcal{K}$. Arranging all the operators of $\mathcal{K}$ in the following manner :

$$
\begin{array}{cccccc}
1 & S_{2} & S_{3} & S_{4} & \cdots & S_{m} \\
T_{2} & S_{2} T_{2} & S_{3} T_{2} & S_{4} T_{2} & \cdots & S_{m} T_{2} \\
\ldots \ldots \ldots \ldots \ldots \ldots \ldots \ldots \ldots \ldots \ldots & \ldots \ldots \ldots . \\
T_{n} & S_{2} T_{n} & S_{3} T_{n} & S_{4} T_{n} & & S_{m} T_{n}
\end{array}
$$

(the first row being composed of the operators of $m$ ) we proceed to prove that there is one and only one operator whose order is prime to $m$ in each row. If $m$ contains just $k^{\prime}$ operators that are commutative with each operator of $m$ there must be just $k^{\prime}$ operators in each one of the given rows that are commuta- 
tive with each operation of $m$; for if $T_{a}$ transforms the operators of the first row according to a given substitution there must be $k^{\prime}$ operators in the first row which transform all the operators of this row according to the inverse of this substitution. These $k^{\prime}$ operators multiplied into $T_{a}$ give the required $k^{\prime}$ operators (of the row which contains $T_{a}$ ) that are commutative with each operator of $m$. Suppose $T_{a}$ is one of these $k^{\prime}$ operators and that $\gamma$ is the order of the corresponding operator in the quotient group $\varkappa / m$. From

$$
\left(S_{a_{1}} T_{a}\right)^{\gamma}=S_{a_{1}}^{\gamma} T_{a}^{\gamma}
$$

and the fact that $\gamma$ is prime to $m$, it follows that we obtain all the operators of $m$ by raising all the operators of the row which contains $T_{a}$ to the $\gamma$ power. This proves that there is one and only one operator whose order is prime to $m$ in each row and that the order of this operator is the same as that of the corresponding operator in $\varkappa / m$. Hence it follows directly from theorem $I$ that $\mathcal{K}$ is the direct product of $m$ and the subgroup of order $n$.

For use in the proof of theorem III one notices that the second hypothesis of theorem III is fulfilled if the operators $M$ of the group $m$ are individually selfconjugate under any certain $n$ extenders of $m$ to $\mathcal{K}$.

Theorem III.-If the order of a solvable group if is $h=m p^{a}$ (where $p$ is a prime and $a$ and $m$ are integers, $m$ being prime to $p$ ) and if all the conjugates under is of every operator $A$ of a subgroup a of order $p^{a}$ are conjugates of $A$ under $a$, then 4 is the direct product of $a$ and a subgroup $n$ of order $m$, and further of contains certain self-conjugate subgrouips $n_{\gamma}$ of the orders $n_{\gamma}=m p^{\gamma}(\gamma=0,1, \ldots, a-1)$.

Each one of the self-conjugate subgroups of $a$ is clearly also a self-conjugate .subgroup of 24 . Hence there must be a $p^{\beta}, 1$ ( $\beta$ being one of the numbers $1,2, \ldots, a-1)$ isomorphism between $\mathcal{L}$ and each one of a series of groups

$$
g_{a-1}, g_{a-2}, g_{a-3}, \cdots, g_{0}
$$

whose orders are divisible by $p^{a-1}, p^{a-2}, p^{a-3}, \cdots, p^{0}$ respectively but by no higher power of $p$. According to theorem II, $q_{1}$ is the direct product of its subgroups of orders $p$ and $m$. Suppose that $g_{\beta}(\beta<a-1)$ is the direct product of its subgroups of orders $p^{\beta}$ and $m$. To the subgroup of order $m$ in $g_{\beta}$ there must correspond a subgroup of order $p m$ in $g_{\beta_{+1}}$. From theorem II it follows that this subgroup of order $p m$ is the direct product of its subgroups of orders $p$ and $m . \quad G_{\beta+1}$ must therefore be the direct product of its subgroups of orders $p^{\beta+1}$ and $m$ whenever $g_{\beta}$ is the direct product of its subgroups of orders $p^{\beta}$ and $m$. Since $g_{1}$ is the direct product of its subgroups of orders $p$ and $m$ it 
follows from what has just been proved that $\&$ must be the direct product of its subgroups of orders $p^{a}$ and $m$. The remainder of the theorem follows directly from this property.

Every group of order $2 m, m$ being any odd number, contains a self-conjugate subgroup of order $m .^{*}$ If it contains a self-conjugate subgroup of order 2 it is evidently the direct product of these two self-conjugate subgroups.

If a group is the direct product of two subgroups its group of cogredient isomorphisms is the direct product of the groups of cogredient isomorphisms of these two subgroups. $\dagger$ The converse of this is not generally true but we may readily prove that it is true if the conditions mentioned in the following theorem are satisfied.

TheOREM IV.-If the group of cogredient isomorphisms $\left(\mathrm{g}^{\prime}\right)$ of a group $(G)$ is the direct product of two subgroups $(m, n)$ whose orders $(m, n)$ are prime to each other, then $\mathrm{g}$ is also the direct product of two subgroups.

COROLLARY.-If a group (L) has an abelian group of cogredient isomorphisms whose order is not a power of a single prime number then $\mathcal{L}$ is the direct product of two subgroups. $\ddagger$

All the operators of $q$ which are commutative with each one of its operators constitute the subgroup $\left(g_{1}\right)$ of order $g_{1}$ which corresponds to identity of $g^{\prime}$ in the isomorphism of $g$ and $g^{\prime}$. Let

$$
m=p_{1}^{a_{1}} p_{2}^{\alpha_{2}} \cdots, \quad n=q_{1}^{\beta_{1}} q_{2}^{\beta_{2}} \cdots, \quad g_{1}=p_{1}^{a_{1}^{\prime}} p_{2}^{a_{2}^{\prime}} \cdots q_{1}^{\beta_{1}^{\prime}} q_{2}^{\beta_{2}^{\prime}} \cdots r_{1}^{\gamma_{1}} r_{2}^{\gamma_{2}} \cdots
$$

where

$$
p_{1}, p_{2}, \cdots, q_{1}, q_{2}, \cdots, \quad r_{1}, r_{2}, \cdots,
$$

are distinct prime numbers; and let $G_{a}$ be any operator of $\mathcal{G}$ which corresponds to a given operator $M_{a}$ of $m$ in the given isomorphism of $g$ and $g^{\prime}$. $\quad G_{a}^{m_{a}}\left(m_{a}\right.$ being the order of $\left.M_{a}\right)$ must occur in $g_{1}$. If the order of $G_{a}^{m a}$ contains a factor $\left(q_{1}^{\beta_{1}^{\prime \prime}} q_{2}^{\beta_{2}^{\prime \prime}} \cdots r_{1}^{\gamma_{1}^{\prime}} \gamma_{2}^{\gamma_{2}^{\prime}} \cdots\right)$ which is prime to $m, G_{a}^{m_{a}}$ must be the direct product of an operator of $g_{1}$ of order $q_{1}^{\beta_{1}^{\prime \prime}} q_{2}^{\beta_{2}^{\prime \prime}} \ldots r_{1}^{\gamma_{1}^{\prime}} r_{2}^{\gamma_{2}^{\prime}} \ldots$ and an operator of order $p_{1}^{a_{1}} p_{2}^{a_{2}} \ldots$. In this case $g_{1}$ must contain some operator $G_{1}$ such that the order of $\left(G_{1} G_{a}\right)^{m_{a}}$ does not contain any factor that is prime to $m$.

We may therefore assume that to each operator of $m$ in the given isomorphism of $g$ and $g^{\prime}$ there corresponds some operator of $g$ whose order does not involve any factor that is prime to $m$. Hence $g$ contains a subgroup of order $p_{1}^{a_{1}+a_{1}^{\prime}} p_{2}^{a_{2}+a_{2}^{\prime}} p_{3}^{a_{3}+a_{3}^{\prime}} \ldots$ which includes all the operators of $g$ whose orders do not

* Frobenius, Berliner Sitzungsberichte, 1895, p. 180.

† Bulletin of the American Mathematical Society, vol. 5, p. 294, 1899.

$\ddagger$ Cf. BURnside, Theory of Groups of a Finite Order, p. 115, 1897. 
involve any factor that is prime to $m$. This subgroup must be self-conjugate. In exactly the same way we may prove that $q$ contains a self-conjugate subgroup of order $q_{1}^{\beta_{1}+\beta^{\prime}} q_{2}^{\beta_{2}+\beta_{2}^{\prime}} q_{3}^{\beta_{3}+\beta_{3}^{\prime}} \ldots$ which includes all the operators of $q$ whose orders do not involve any factor that is prime to $n$. If each of the $r$ 's is equal to unity $g$ is the direct product of $m$ and $n$. In general $g$ is evidently the direct product of $m, n$ and the subgroup of order $r_{1}^{\gamma_{1}} r_{2}^{\gamma_{2}} r_{3}^{\gamma_{3}} \ldots$ which is contained in $\mathcal{G}$, and hence it is also the direct product of one of these three groups and the product of the other two.

If a group $m$ contains only identity as a self-conjugate operator, then it is simply isomorphic to its group of cogredient isomorphisms. If moreover $\mathrm{m}$ is a subgroup of a group $\mathcal{K}$ with the property of the hypothesis of theorem II, then $\mathcal{K}$ is the direct product of $\mathrm{m}$ and a subgroup which is simply isomorphic to $\varkappa / m$. This subgroup is composed of all the operators of $\mathcal{K}$ which are commutative with every operator of $m$. In fact, it is evident that all the operators of a group which are commutative with every operator of any one of its self-conjugate subgroups must always constitute a self-conjugate subgroup. This subgroup may evidently be the entire group. In particular, when a complete group is self-conjugate in any group the latter is the direct product of this complete group and the corresponding quotient group.*

If $g$ is the direct product of $g_{1}, g_{2}$ then any group contained in $g_{1}$ together with any group contained in $g_{2}$ generates a group which is the direct product of these two generating groups. Suppose $g$ may be represented as a primitive substitution group. Then the subgroup which includes all its substitutions that do not involve a given element is maximal and not self-conjugate. From this it follows that this subgroup does not contain any self-conjugate subgroup of $g$ besides identity. Hence the given subgroup must be formed by establishing a simple isomorphism between the substitution group which are simply isomorphic to $g_{1}$ and $g_{2}, i$. e., the necessary and sufficient condition that $g$ can be represented as a primitive substitution group is that $\mathrm{g}_{1}$ and $\mathrm{g}_{2}$ are simply isomorphic simple groups of a composite order. $\dagger$ When this condition is satisfied c can evidently be represented as a primitive group in only one way and the degree of this primitive group is the square root of the order of $\mathrm{g} \cdot \downarrow$

From the preceding paragraph it follows directly that the degrees of the primitive groups which are the direct products of two groups have a $(1,1)$ correspondence to the simple groups of composite order; the first group of this kind being of degree 60 and order 3600, the second of degree 168 and order 28224,

* Hölder, Mathematische Annalen, vol. 46, p. 325.

†Cf. Burnside, Theory of Groups, p. 190, 1897.

$\ddagger$ Cf. Maillet, These de Doctorat, p. 31, Paris, 1892. 
etc. These groups can be represented in a large number of different ways as imprimitive groups.

If $g_{1}$ and $g_{2}$ are the direct products of $a$ and $\beta$ groups respectively then $g$ may be said to be the direct product of these $a+\beta$ groups and it can be represented as the product of any $a+\beta$ transitive substitution groups (written in distinct elements) which satisfy the condition that they are simply isomorphic to all the given $a+\beta$ groups, each being associated with only one of them. Hence the necessary and sufficient condition that $q$ is the direct product of $\gamma$-groups is, that it contains $\gamma$ self-congugate subgroups $\left(g_{1}, g_{2}, \cdots, g_{\gamma}\right)$ such that the group generated by any $\delta$ of them has only identity in common with any one of the remaining $\gamma-\delta$ and that the order of $g$ is the product of the orders of these subgroups.* When $\gamma>2$ it is clearly impossible to represent $G$ as a primitive substitution group. Hence the necessary and sufficient condition that a direct product may be represented as a primitive substitution group is that it contains just two factors and that these are simply isomorphic simple groups of composite order.

Cornell University.

* HöLDER, Mathematische Annalen, vol. 43, p. 330, 1893. 